\title{
Error analysis for time-interleaved Analog Channels
}

\author{
Baiying Y $u^{1,2}$, William C. Black, Jr. ${ }^{1}$ \\ ${ }^{1}$ Analog and Mixed-Signal Design Center \\ Department of Electrical and Computer Engineering \\ Iowa State University, Ames, IA 50011-3060 \\ ${ }^{2}$ Agere Systems, Allentown, PA 18103
}

\begin{abstract}
Time-interleaved architectures are frequently employed in highspeed data converters and signal processing channels in order to achieve the highest possible speed. However, the performance of the combined channels is reduced if each channel is not well matched in terms of gain. converter offset and timing skew. In this paper, the errors introduced by gain and offset mismatch and timing skew is analyzed in detail. The digital spectra due to mismatch between channels is derived by applying digital signal processing theories and the closed-forms of signal-to-noise ratio (SNR) are derived and verified using Matlab ${ }^{\mathrm{TM}}$.
\end{abstract}

\section{INTRODUCTION}

High-speed data conversion and signal processing circuits are demanded for data communication. data storage. etc. In the extremes of speed. exotic technologies must be used to achieve data rates beyond those obtained with a conventional silicon implementation [1]-[3]. By using time-interleaved architecture, the maximum conversion rate practical with any technology is extended by the use of an array of identical channels. This topology applied to an analog-to-digital converter is shown in Figure 1 .

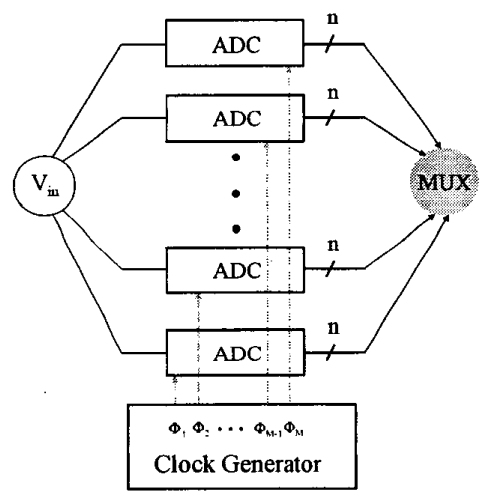

Figure 1 Generic scheme of time-interleaved A/D architecture

Basically, each individual channel is running at a speed of $\mathrm{f}_{\mathrm{ck}}$ but at different clock phases which are generated by the multi-phase clock generator. The phase difference between adjacent clocks should be the same, otherwise, timing skew will introduce extra error into the system. For the case shown in Figure 1 where an array of $\mathrm{A} / \mathrm{D}$ converters are employed, the outputs from the array of $M$ converters can be combined by a high speed ' $M U X$ ', resulting in a high speed $A D C$ with a sampling speed of $M x f_{c k}$ [4]-[6]. This technique trades off increased die area for increased speed in a nearly one for one relationship but at reduced performance if the channels are not well matched in terms of gain, offset and sampling skew [7]. In the following sections, the digital spectra due to mismatch between channels in terms of gain. converter offset and timing skew are derived and demonstrated. The closed-forms of the signal-to-noise ratio are derived and verified by Matlab ${ }^{\mathrm{TM}}$ simulation.

\section{ERROR ANALYSIS}

\subsection{Timing skew}

The digital spectrum, $F(\omega)$, of the sampled data by timeinterleaved architecture can be represented by [8]

$$
F(\omega)=\frac{1}{M T} \sum_{m=0}^{M-1}\left[\sum_{k=-\infty}^{\infty} F^{a}\left[\omega-k\left(\frac{2 \pi}{M T}\right)\right] e^{j[\omega-k(2 \pi / M T)] t_{m}}\right] e^{-j m \omega T}
$$

$F^{a}(\omega)$--- the Fourier Transform of $f(t)$

$\mathrm{M}$--- number of interleave

$\mathrm{T}$--- period of the whole array combining all single channels $\mathrm{t}_{\mathrm{m}}$--- real sampling time for $\mathrm{m}^{\text {th }}$ channel

$t_{m}=m T-r_{m} T$

Substitute (2) into (1), we have

$$
\begin{aligned}
F(\omega) & \left.=\frac{1}{M T} \sum_{m=0}^{M-1}\left[\sum_{k=-\infty}^{\infty} F^{a}\left(\omega-k \frac{2 \pi}{M T}\right)\right)^{-j\left(\omega-k \frac{2 \pi}{M T}\right) r_{m} T} e^{-j k m \frac{2 \pi}{M}}\right] \\
& =\frac{1}{T} \sum_{k=-\infty}^{\infty} A(k) F^{a}\left(\omega-k \frac{2 \pi}{M T}\right)
\end{aligned}
$$

where

$A(k)=\sum_{m=0}^{M-1}\left[\frac{1}{M} e^{-j r_{m} 2 \pi f_{i n} / f_{s}}\right] e^{-j k m 2 \pi / M}$

For a given sine wave $f(t)=\sin \left(\omega_{i n} t\right)$. the Fourier transform is given by

$F^{a}(\omega)=\pi\left[\delta\left(\omega+\omega_{i n}\right)-\delta\left(\omega-\omega_{i n}\right)\right]$

Substitute (5) into (3), we have 


$$
\begin{array}{r}
F(\omega)=\frac{1}{T} \sum_{k=-\infty}^{\infty}\left[A(k) 2 \pi \delta\left(\omega+\omega_{i n}-k \frac{2 \pi}{M T}\right)\right. \\
\left.+B(k) 2 \pi \delta\left(\omega-\omega_{i n}-k \frac{2 \pi}{M T}\right)\right]
\end{array}
$$

where

$$
\begin{aligned}
& A(k)=\frac{-1}{2 j} \sum_{m=0}^{M-1}\left[\frac{1}{M} e^{j r_{m} 2 \pi f_{i n} / f_{s}}\right] e^{-j k m(2 \pi / M)} \\
& B(k)=\frac{1}{2 j} \sum_{m=0}^{M-1}\left[\frac{1}{M} e^{-j r_{m} 2 \pi f_{i n} / f_{s}}\right] e^{-j k m(2 \pi / M)}
\end{aligned}
$$

It is very easily to prove that

$$
A(k)=-B^{*}(M-k)
$$

From equation (6), we can observe that the digital spectrum comprises $M$ pairs of line spectra, each pair centers at the fractional of the sampling frequency of $\mathrm{f}_{\mathrm{s}}$, such as $f_{s} / M$, $2 f_{s} / M, \ldots,(M-1) f_{s} / M$. The digital spectrum of a four-way, timeinterleaved array with timing skew is shown Figure 2 along with the amplitude for each component.

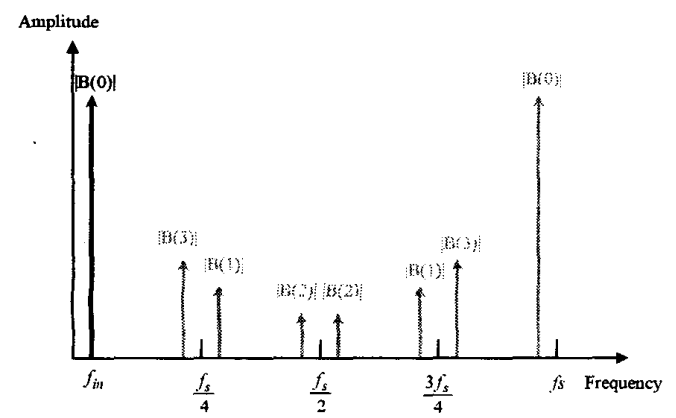

Figure 2 Digital spectrum of a four-way, time-interleaved array with timing skew

By Parseval's theorem, we have

$$
\begin{aligned}
& P_{1}=\sum_{k=0}^{M-1}|A(k)|^{2}=\frac{1}{4} \\
& P_{2}=\sum_{k=0}^{M-1}|B(k)|^{2}=\frac{1}{4}
\end{aligned}
$$

The total signal and distortion power is

$$
P=P_{1}+P_{2}=\frac{1}{2}
$$

Assume $r_{\mathrm{m} 1}$ is normally distributed with standard deviation of $\sigma_{\mathrm{t}}$ and zero mean. The signal power located at $\omega_{0}$ is

$$
\begin{aligned}
|B(0)|^{2} & =\left|\sum_{m=0}^{M-1} \frac{1}{2 M j} e^{-j r_{m} 2 \pi f i n / f s}\right|^{2} \\
& \approx \frac{1}{4} \mid \sum_{m=0}^{M-1} \frac{1}{M}\left(1+j r_{m} 2 \pi f_{i n} f_{s}-\left.\frac{1}{2}\left(r_{m n} 2 \pi f_{i n} / f_{s}\right)^{2}\right|^{2}\right. \\
& =\frac{1}{4}\left|1-\frac{1}{2}\left(2 \pi f_{i n} / f_{s}\right)^{2} \sigma_{t}{ }^{2}\right|^{2}
\end{aligned}
$$

The noise power in the range of $\left(0.1 /\left(2 f_{s}\right)\right)$ should be

$$
\begin{aligned}
\text { noise }=\left(P-2|B(0)|^{2}\right) / 2 & =\frac{1}{4}-\frac{1}{4}\left(1-\frac{1}{2}\left(2 \pi \sigma_{t} f_{i n} / f_{s}\right)^{2}\right)^{2} \\
& \approx\left(2 \pi \sigma_{\mathrm{t}} f_{\text {in }} / f_{s}\right)^{2}
\end{aligned}
$$

The signal to noise ratio can be obtained

$$
\begin{aligned}
S N R & =10 \log 10\left(\frac{|B(0)|^{2}}{\text { noise }}\right) \\
& \approx\left(20 \log 10 \frac{1}{2 \pi \sigma_{t} f_{\text {in }} / f_{s}}\right)(d B)
\end{aligned}
$$

From equation (15), we can easily observe that the signal-to-noise ratio (SNR) is a function of $\sigma_{t} f_{i n} / f_{s}$. If $\sigma_{t} f_{i n} / f_{s}$ doubles, the SNR will decrease about $6 \mathrm{~dB}$.

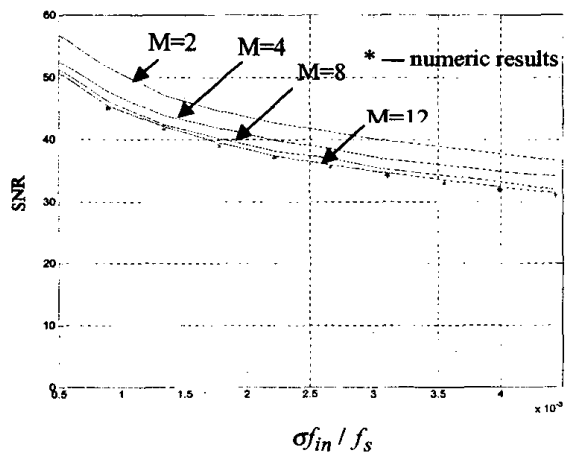

Figure 3 The relation between SNR and timing skew

Figure 3 shows the comparison between the numeric results using equation (15) and discrete time simulation results. The simulated results were obtained by averaging 100 channels using Matlab. As we can see, the higher the time-interleaved number $M$, the smaller the error between the numeric results and the simulated results. For interleave numbers greater than 12. equation (15) can be used to estimate the SNR very precisely. For a four-way, timeinterleaved array, the SNR can be estimated using the following equation.

$$
S N R=20 \log 10\left(\frac{1}{2 \pi \sigma_{i n} / f_{s}}\right)+3(d B)
$$

Hence, in order to achieve $6 \mathrm{~b}$ accuracy at the Nyquist rate with a sampling frequency of $500 \mathrm{MS} / \mathrm{s}$, the timing skew between the different channels needs to be smaller than $12 \mathrm{ps}$ based on equation (16).

\subsection{Gain Mismatch}

Gain mismatch can be modeled by making the amplitude of the input signals sampled be different for different channels. Assume for each individual channel that the analog input signal is $A_{m} \sin \left(\omega_{i n} t\right)$, where $\mathrm{m}=0, \ldots \mathrm{M}-1$. Based on equation (3), we can derive the digital spectrum of time-interleaved array with gain mismatch. For a sine wave $A_{m} \sin \left(\omega_{i n} t\right)$, the Fourier transform is given by

$F_{m}{ }^{a}(\omega)=j \pi A_{m}\left[\delta\left(\omega+\omega_{i n}\right)-\delta\left(\omega-\omega_{i n}\right)\right]$ 
where $m=0,1, \ldots \mathrm{M}-1$

If we assume that there is no timing error, $r_{w}$ is equal to zero. Substituting (17) into (3), we get

$F(\omega)=\frac{1}{T} \sum_{k=-\infty}^{\infty} A(k)\left[2 \pi \delta\left(\omega+\omega_{i n}-k \frac{2 \pi}{M T}\right)-2 \pi \delta\left(\omega-\omega_{i n}-k \frac{2 \pi}{M T}\right)\right]$

where

$A(k)=\frac{j}{2 M} \sum_{m=0}^{M-1} A_{m} e^{-j k m \frac{2 \pi}{M}}$

As we can see from equation (18), the digital spectrum with gain mismatch also comprises $M$ pairs of line spectra that centers at the fraction of the sampling frequency $f_{s}$, that is the same as that with timing skew. However, the distortion introduced by the gain mismatch has the same magnitude for each pair, while this is not true for errors introduced by timing skew. The digital spectrum of a four-way, time-interleaved array is shown in Figure 4.

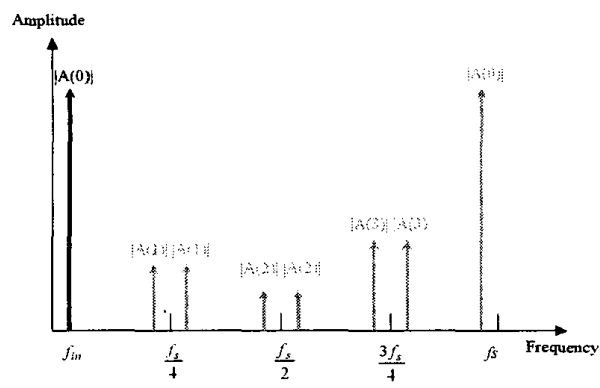

Figure 4 Digital spectrum of a four-way, time-interleaved array with gain mismatch

Assume $A_{w}$ is normally distributed with standard deviation of $\sigma_{A}$ and mean of $\bar{A}$. By Parseval's theorem, we have

$$
\begin{aligned}
& P_{1}=\sum_{k=0}^{M-|A(k)|^{2}}=M \sum_{k=0}^{M-1}\left(\frac{A_{m}}{2 M}\right)^{2} \approx \frac{1}{4}\left(\sigma_{A}{ }^{2}+\bar{A}^{2}\right) \\
& P_{2}=\sum_{k=0}^{M-1}|A(k)|^{2} \approx \frac{1}{4}\left(\sigma_{A}{ }^{2}+\bar{A}^{2}\right)
\end{aligned}
$$

The total signal and distortion power is

$$
P=P_{1}+P_{2}=\frac{1}{2}\left(\sigma_{. t}^{2}+\bar{A}^{2}\right)
$$

The signal power of the fundamental is

$$
|A(0)|^{2}=\left|\frac{1}{2 M} \sum_{k=0}^{M-1} A_{m}\right|^{2} \approx\left|\frac{1}{2} \hat{A}\right|^{2}=\frac{1}{4} \bar{A}^{2}
$$

The power of the noise in the range of $\left(0,1 /\left(2 f_{s}\right)\right)$ is

$$
\text { noise }=\left(P-2|A(0)|^{2}\right) / 2=\frac{1}{4} \sigma_{A}{ }^{2}
$$

Assume the gain $a_{m}$ for each converter is proportional to $A_{m}$ with constant efficient $\beta$, then

$a_{m}=\beta A_{m}$

where $m=0 . . . M-1$

So. we have $\sigma_{a}=\beta \sigma_{A}$

$\bar{a}=\bar{A}$

Then, the signal-to-noise ratio is

$S N R=10 \log 10\left(\frac{\frac{1}{4} \bar{A}^{2}}{\frac{1}{4} \sigma_{A}{ }^{2}}\right)=20 \log 10\left(\frac{\bar{A}}{\sigma_{A}}\right)=20 \log 10\left(\frac{\bar{a}}{\sigma_{a}}\right)$ (27)

From equation (27), we can see that, if the gain mismatch can be decreased by two times, the SNR can be increased by about $6 \mathrm{dH}$.

Figure 5 shows the comparison between the numeric results obtained based on equation (27) and the simulated results obtained by averaging 100 channels. As we can see from Figure 5 , the numeric results are very close to the simulated results when the time-interleaved number $\mathrm{M}$ is greater than 12 .

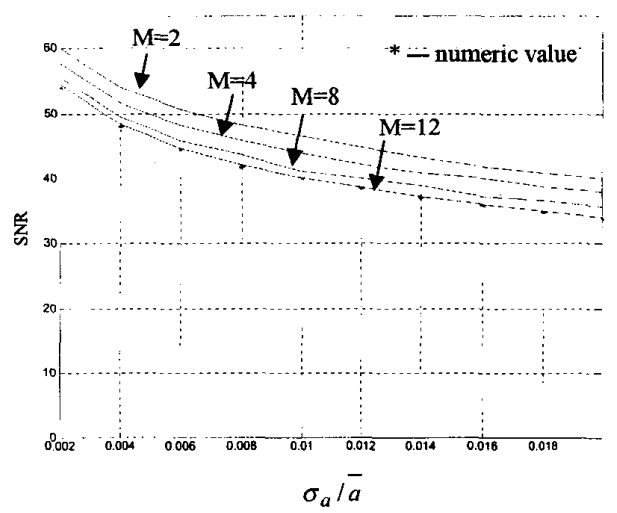

Figure 5 The relation between SNR and gain mismatch

For a four-way, time-interleaved array, the signal to noise ratio can be estimated using the following equation.

$S N R=20 \log 10\left(\frac{\bar{a}}{\sigma_{a}}\right)+3(d B)$

In order to achieve $6 \mathrm{~b}$ accuracy, the gain mismatch $\sigma_{a / \bar{a}}$ between channels needs to be smaller than 0.018 .

\subsection{Converter Offset Mismatch}

Converter offset mismatch can be modeled by simply adding a DC level with the input signal that is unique for each channel. Assume for each individual channel that the analog input signal is $A \sin \left(\omega_{i n} t\right)+D_{m}$. Then. the digital spectrum for the composite time-interleaved array can be derived. For an input signal $A \sin \left(\omega_{i n} t\right)+D_{m}$, the Fourier transform is given by

$F^{a}(\omega)=A j \pi\left[\delta\left(\omega+\omega_{i n}\right)-\delta\left(\omega-\omega_{i n}\right)\right]+2 \pi D_{m} \delta(\omega)$

Substituting (29) into (3), we get

$$
\begin{aligned}
F(\omega)= & \frac{1}{T} \sum_{k=-\infty}^{\infty} A j \pi\left[\delta\left(\omega+\omega_{i n}-k \frac{2 \pi}{T}\right)-\delta\left(\omega-\omega_{i n}-k \frac{2 \pi}{T}\right)\right] \\
& +\frac{1}{T} \sum_{k=-\infty}^{\infty} D(k) 2 \pi \delta\left(\omega-k \frac{2 \pi}{M T}\right)
\end{aligned}
$$


$D(k)=\frac{1}{M} \sum_{m=0}^{M-1} D_{m} e^{-j k m \frac{2 \pi}{M}}$

It is easily to prove that

$|D(k)|=\left|D^{*}(M-k)\right|=|D(M-k)|$

The first term in (30) is the well-known digital spectrum representation of a uniformly sampled sine wave signal [9]. The second term is the spectra due to channel offset mismatch. As we can see from equation (30), the spectra lines due to channel offset mismatch are located at fractions of the overall sampling frequency. Figure 6 shows the digital spectrum of a four-way, time-interleaved array with channel offset.

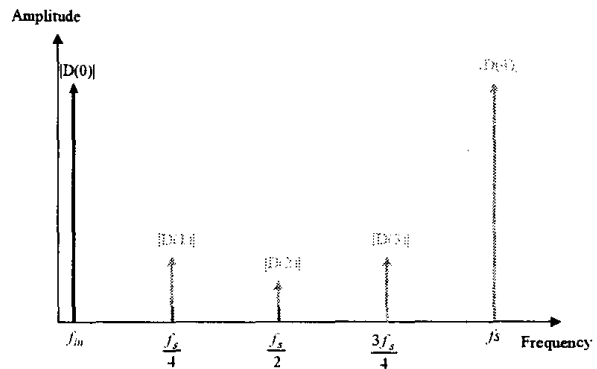

Figure 6 Digital spectrum due to channel offset mismatch

Assume $D_{m}$ is normally distributed with standard deviation of $\sigma_{D}$ and zero mean. The power of the noise in the range of $\left(0.1 /\left(2 f_{s}\right)\right)$ is

noise $=\frac{1}{2} \sum_{k=0}^{M-1} D(k)=\frac{M}{2} \sum_{m=0}^{M-1}\left(\frac{D_{m}}{M}\right)^{2}=\frac{\sum_{m=0}^{M-1} D_{m}{ }^{2}}{M} \approx \sigma_{D}{ }^{2}$

The signal power is

signal $=\frac{A^{2}}{4}$

The signal-to-noise ratio is

$S N R=10 \log ($ signal $/$ noise $)=20 \log 10\left(\frac{A}{\sqrt{2} \sigma_{D}}\right)$

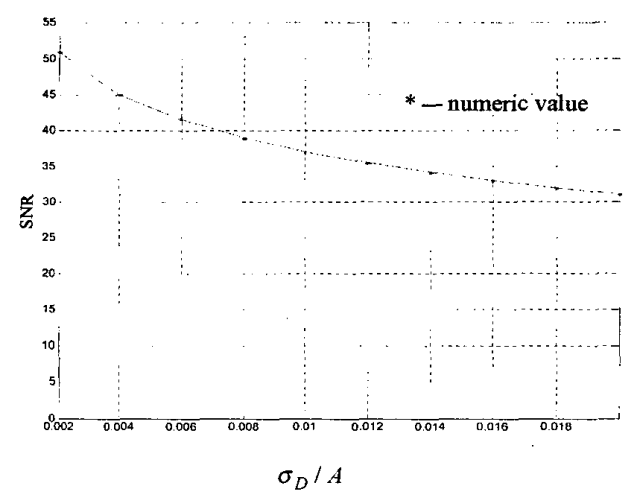

Figure 7 The relation between SNR and converter offset mismatch
Equation (34) implies that if the amplitude of the analog input signal is increased by 2 , or the channel mismatch is decreased by 2 , the SNR can be improved by about $6 \mathrm{~dB}$.Figure 7 shows the comparison between numeric results obtained by using equation (34) and the simulated results obtained by averaging 100 channels using Matlab. Different from the previous two cases, the signal-to-noise ratio is not dependent on the number of interleaved channels. As we can see from Figure 7 , the curve fit of the numeric results match the simulated results.

\section{CONCLUSION}

By applying digital signal processing theories, the digital spectra of non-ideal time-interleaved analog channels are derived and demonstrated. From Matlab simulations of time-interleaved channels with timing skew and gain mismatch, it can be seen that the SNR slightly depends on the interleaved number $M$ if $M$ is less than 12. The curve fit of the closed-forms of the SNR matches the simulation results when the interleaved number is greater than 12 for time-interleaved analog channels with timing skew and gain mismatch. For time-interleaved channeis with converter offset, the signal-to-noise ratio is independent of the interleave number $\mathrm{M}$. Based on the closed-forms provided. it is very easy to derive the matching requirements in order to meet design specifications.

\section{REFERENCES}

[1] Xiao. P. et al., "A $4 b$ 8Gsample/s $A / D$ converter in SiGe bipolar technology". ISSCC Digest of Technical papers, pp. 124125, Feb. 1997

[2] Ducourant, T., " $3 G H z, 150 \mathrm{~mW}, 4$ Bit GaAs Analog to Digital Converter", Technical Digest - GaAs IC symposium, pp. 209-212, 1992

[3] Ducourant, T., " $A$ bit, $2.2 \mathrm{Gs} / \mathrm{s}$ monolithic A/D converter with gigahertz bandwidth. and 6 bit A/D converter system", Technical Digest - GaAs IC Symposium. pp. 337-340, 1989

[4] ] William C. Black, Jr.. David A. Hodges, "Time Interleaved Converter Arrays". IEEE Journal of Solid State Circuits, Vol. SC15. no. 6. pp. 1022-1029, pp. 962-970. Dec. 1980

[5] Ken Purlton et al., "A 1-GHz 6-bit ADC System", IEEE Journal of Solid-State Circuits, Vol. SC-22, no. 6, pp. . Dec. 1987

[6] Daihong Fu. et al.," A Digital Background Calibration Technique for Time-Interleaved Analog-to-Digital Converters", IEEE Journal of Solid-State Circuits. Vol. 33, no. 12. pp 19041911. Dec. 1998

[7] William C. Black. Jr.. “ High Speed CMOS A/D Converter Techniques". Ph.D Dissertation. University of Berkeley, Nov.. 1980

[8] Jenq. Y. C.. " Digital spectra of nonuniformly sampled signals: Fundamentals and high speed waveform digitizers", IEEE Transactions on Instrumentation and measurement. Vol. 39, No. 1, Feb.. pp. 245-251. 1990

[9] Abraham Peles. Bede Liu, "Digital Signal Processing. theory, design, and implementation", John Wiley \& Sons. 1976 